\title{
Impact of endplate-implant area mismatch on rates and grades of subsidence following stand-alone lateral lumbar interbody fusion: an analysis of 623 levels
}

\author{
Nitin Agarwal, MD, Michael D. White, BS, Xiaoran Zhang, MD, Nima Alan, MD, Alp Ozpinar, MD, \\ David J. Salvetti, MD, Zachary J. Tempel, MD, David O. Okonkwo, MD, PhD, Adam S. Kanter, MD, \\ and D. Kojo Hamilton, MD
}

Department of Neurological Surgery, University of Pittsburgh Medical Center, Pittsburgh, Pennsylvania

\begin{abstract}
OBJECTIVE Stand-alone lateral lumbar interbody fusion (LLIF) is a useful minimally invasive approach for select spinal disorders, but implant subsidence may occur in up to $30 \%$ of patients. Previous studies have suggested that wider implants reduce the subsidence rate. This study aimed to evaluate whether a mismatch of the endplate and implant area can predict the rate and grade of implant subsidence.
\end{abstract}

METHODS The authors conducted a retrospective review of prospectively collected data on consecutive patients who underwent stand-alone LLIF between July 2008 and June 2015; 297 patients (623 surgical levels) met inclusion criteria. Imaging studies were examined to grade graft subsidence according to Marchi criteria. Thirty patients had radiographic evidence of implant subsidence. The endplates above and below the implant were measured.

RESULTS A total of 30 patients with implant subsidence were identified. Of these patients, 6 had Marchi grade 0,4 had grade I, 12 had grade II, and 8 had grade III implant subsidence. There was no statistically significant correlation between the endplate-implant area mismatch and subsidence grade or incidence. There was also no correlation between endplate-implant width and length mismatch and subsidence grade or incidence. However, there was a strong correlation between the usage of the 18-mm-wide implants and the development of higher-grade subsidence $(p=0.002)$ necessitating surgery. There was no significant association between the degree of mismatch or Marchi subsidence grade and the presence of postoperative radiculopathy. Of the 8 patients with $18-\mathrm{mm}$ implants demonstrating radiographic subsidence, $5(62.5 \%)$ required reoperation. Of the 22 patients with 22-mm implants demonstrating radiographic subsidence, $13(59.1 \%)$ required reoperation.

CONCLUSIONS There was no correlation between endplate-implant area, width, or length mismatch and Marchi subsidence grade for stand-alone LLIF. There was also no correlation between either endplate-implant mismatch or Marchi subsidence grade and postoperative radiculopathy. The data do suggest that the use of 18 -mm-wide implants in standalone LLIF may increase the risk of developing high-grade subsidence necessitating reoperation compared to the use of 22-mm-wide implants.

https://thejns.org/doi/abs/10.3171/2020.1.SPINE19776

KEYWORDS lateral lumbar interbody fusion; lateral access; subsidence; stand-alone

$\mathrm{T}$ $\checkmark$ HE lateral lumbar interbody fusion (LLIF) procedure is a retroperitoneal transpsoas approach to gain access to the lumbar disc space for graft implantation. It has been shown to be effective for surgical correction of a multitude of spinal disorders. ${ }^{8,11,16}$ The LLIF approach has grown in popularity as an alternative to both anterior and posterior access channels, as it avoids requiring an access surgeon. Furthermore, LLIF limits excessive retraction that can damage viscera or vascular structures seen when using the anterior approach or the higher risk of dural tear, muscle denervation, and increased risk of infections seen when using the posterior approach. ${ }^{6,11,17,18}$ It is also advantageous in that it preserves the anterior and posterior annular/ligamentous structures.

Despite the advantages of the LLIF procedure, it is not without its own complications. Graft subsidence is of par-

ABBREVIATIONS BMI = body mass index; DEXA = dual-energy $\mathrm{x}$-ray absorptiometry; LLIF = lateral lumbar interbody fusion; PI-LL = pelvic incidence-lumbar lordosis. SUBMITTED June 30, 2019. ACCEPTED January 2, 2020.

INCLUDE WHEN CITING Published online March 6, 2020; DOI: 10.3171/2020.1.SPINE19776. 
ticular concern, with the indirect decompression of neural elements, as a loss of disc height can increase stenosis, sagittal malalignment, and the risk of adjacent-segment disturbances. ${ }^{13}$ Progressive deformity and neural compression as a result of subsidence can increase the chances of failed fusion and the need for reoperation. ${ }^{3,7,10}$ Rates of subsidence in patients undergoing stand-alone LLIF have been reported to range from $10 \%$ to $30 \% .^{10,13}$ Previous studies have demonstrated that the use of wider implants is associated with lower incidences of subsidence, ${ }^{9,10,13}$ but there is a paucity of studies investigating the effects of endplate-implant area mismatch on graft subsidence. This study aimed to determine whether endplate-implant area mismatch or implant size in stand-alone LLIF is associated with higher grades and rates of subsidence postoperatively.

\section{Methods}

A retrospective review of prospectively collected data was conducted on consecutive patients who underwent stand-alone LLIF at a single institution between July 2008 and June 2015. A total of 297 patients (623 levels) met inclusion criteria. In patients undergoing LLIF at our institution, we obtain images at standard follow-up times, including anteroposterior plain radiographs immediately postoperatively and at the 6-week, 3-month, 6-month, 1-year, and 2-year follow-up visits. Additionally, CT scans of the lumbar spine are obtained immediately postoperatively and at the 1-year follow-up visit. The postoperative CT scan was reviewed to ensure that the endplates had not been violated intraoperatively. Images were evaluated by the surgical team and graded according to the Marchi criteria. ${ }^{13}$

Subsidence grading was based on the percentage of disc space or vertebral body collapse around the interbody graft compared to that of the immediate postoperative imaging: Marchi grade 0,0\%-24\% collapse; grade I, 25\%$49 \%$ collapse; grade II, $50 \%-74 \%$ collapse; and grade III, $75 \%-100 \%$ collapse. Low-grade subsidence was defined as grade 0 or grade I, whereas high-grade subsidence was defined as either grade II or grade III. Thirty-four patients had radiographic evidence of implant subsidence; however, 4 patients were excluded because exact graft size measurements from the manufacturer were not available, yielding a study cohort of 30 patients. Consequently, 30 patients without evidence of radiographic subsidence were selected and paired with patients from the subsidence group undergoing LLIF at the same vertebral levels to create a matched control group. The control group was appropriately matched for sex and levels operated on. Furthermore, the control patients were selected to match age, body mass index (BMI), smoking status, bone density, and pelvic incidence-lumbar lordosis (PI-LL) mismatch as closely as possible. The endplates above and below the implant were measured to determine the endplate-implant area mismatch. Figure 1 demonstrates how the area of the vertebral endplates and interbody grafts was calculated. The mismatch between the endplate and the interbody graft is calculated as follows: mismatch = endplate area - graft area, with a positive number indicating the area of the endplate is larger than the area of the graft. The mismatch will hereby be denoted as "superior endplate mismatch/inferior endplate mismatch" to demonstrate the mismatch above and below the graft, respectively.

Two-way ANOVA was performed to determine the statistical relationship between the area mismatch of the upper endplate and lower endplate and subsidence grade. Chi-square analysis was used for the analysis of subsidence grade and reoperation rates, implant size, presence of postoperative radicular symptoms, smoking status, and sex (GraphPad). A binary logistical regression analysis was used to investigate any statistical relationship between the degree of endplate-implant mismatch and the presence of postoperative radiculopathy (SPSS Inc.).

\section{Results}

Of the 297 patients included in the study, a total of 34 patients $(11.4 \%)$ undergoing LLIF were found to have radiographic implant subsidence. Thirty of these patients had sufficient records regarding manufacturer graft size and were included in the analysis. The subsidence and control groups were both made up of 4 males (13\%) and 26 females $(87 \%)$. The mean ages were 66.1 and 65.5 years for the subsidence and control groups, respectively. The mean follow-up durations were 38.3 months for the subsidence group and 40.3 months for the control group. The subsidence group was found to have a lower bone density based on dual-energy x-ray absorptiometry (DEXA) $\mathrm{t}$-scores when compared to the control group $(\mathrm{p}=0.016)$. All other demographic variables did not significantly differ between the two cohorts (Table 1).

The indications for surgery included degenerative scoliosis, degenerative disc disease with or without foraminal stenosis, low-grade spondylolisthesis, adjacent-segment disease following prior fusion, and far-lateral disc herniation. A total of 30 patients undergoing LLIF were identified as having radiographic implant subsidence, with a mean of 4.7 months from the time of surgery to the development of subsidence. Of these patients, 6 had Marchi grade 0,4 had grade I, 12 had grade III, and 8 had grade III implant subsidence. Patients with grade 0 subsidence had an average superior endplate/inferior endplate-implant area mismatch of $2.69 \mathrm{~cm}^{2} / 1.86 \mathrm{~cm}^{2}$; grade I, 1.21 $\mathrm{cm}^{2} / 2.51 \mathrm{~cm}^{2}$; grade II, $1.47 \mathrm{~cm}^{2} / 1.81 \mathrm{~cm}^{2}$; and grade III, $1.94 \mathrm{~cm}^{2} / 2.28 \mathrm{~cm}^{2}$ (Table 2). There was no statistically significant correlation between endplate-implant area, width, and length mismatch and subsidence grade. The mean superior/inferior endplate-implant area mismatches between the subsidence group and control group were 1.81 $\mathrm{cm}^{2} / 2.04 \mathrm{~cm}^{2}$ and $1.63 \mathrm{~cm}^{2} / 2.00 \mathrm{~cm}^{2}$, respectively. There was also no significant difference between the area, width, and length of mismatch between the groups. Graft height was also not found to be different between the subsidence and control groups and was not associated with the grade of subsidence. Finally, no significant correlation was found between subsidence grade and PI-LL mismatch, DEXA tscores, smoking status, or BMI.

A strong correlation was noted between the use of the 18-mm-wide implants and the development of highergrade subsidence $(p=0.002)$ that necessitated surgery. Only 18- and 22-mm-wide implants were used. Of the 8 


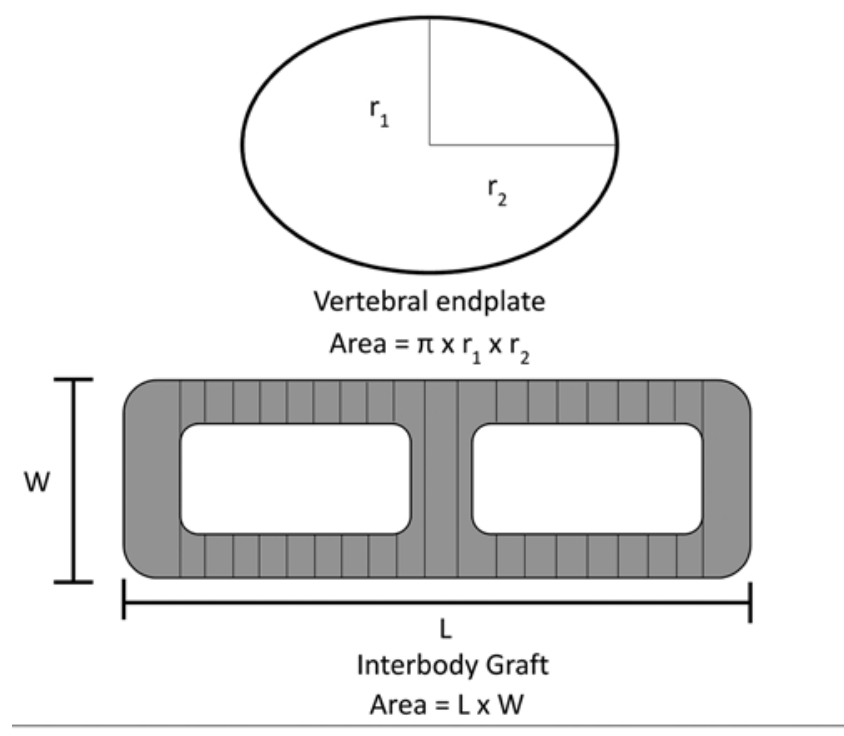

Endplate - Implant Mismatch $=$ Endplate Area - Implant Area

FIG. 1. Calculations utilized to determine the area of the vertebral endplate and interbody graft. Mismatch was defined as the difference between the calculated endplate and implant areas. $L=$ length; $W=$ width.

patients with 18-mm implants demonstrating radiographic subsidence, $5(62.5 \%)$ required reoperation. Of the 22 patients with 22-mm implants demonstrating radiographic subsidence, $13(59.1 \%)$ required reoperation. Indications for reoperation included return or progression of symptoms, new-onset neurological deficits, and/or debilitating back pain. No statistical significance was found between implant size and reoperation rates.

A binary logistical regression analysis was conducted to determine if there was an association between the degree of mismatch of both the superior and inferior endplate widths to the graft width and the presence of postoperative radiculopathy. There was no statistically significant association between the degree of mismatch and the presence of postoperative radiculopathy. Similarly, there was also no statistical significance between Marchi grade and the presence of postoperative radiculopathy on chisquare analysis.

\section{Discussion}

LLIF offers a minimally invasive approach to achieve interbody distraction and fusion of the lumbar spine. An important consideration with stand-alone LLIF is the development of graft subsidence, because endplate collapse can be detrimental to the mechanical stability and it affects the indirect decompression of neural elements. The clinical implications of subsidence in stand-alone LLIF may be more pronounced than those of subsidence in the more traditional posterior and transforaminal approaches given that it does not take advantage of the additional support and load sharing provided by pedicle screws, potentially leading to high rates of reoperation and failed fusion with high-grade subsidence. ${ }^{3,7,10}$ In this retrospective, institutional, 7-year cohort review of those undergoing stand-alone LLIF, endplate-graft area, width, and length
TABLE 1. Demographics of the control and subsidence groups

\begin{tabular}{lcc}
\hline \multicolumn{1}{c}{ Variable } & Control Group & Subsidence Group \\
\hline Age, yrs & $65.53 \pm 1.83$ & $66.13 \pm 1.63$ \\
\hline Male & $4(13 \%)$ & $4(13 \%)$ \\
\hline Female & $26(87 \%)$ & $26(87 \%)$ \\
\hline BMl & $30.21 \pm 1.23$ & $28.23 \pm 1.11$ \\
\hline DEXA t-score & $-0.8^{*}$ & $-1.7^{*}$ \\
\hline Patients who smoke & $11(37 \%)$ & $16(53 \%)$ \\
\hline PI-LL mismatch & $10.4^{\circ}$ & $12.1^{\circ}$ \\
\hline Follow-up, mos & 40.3 & 38.3 \\
\hline
\end{tabular}

Values are presented as the mean $\pm \mathrm{SD}$, as the mean, or as the number (\%) of patients.

* Statistically significant difference noted with a lower mean DEXA t-score among the subsidence group $(p=0.016)$.

mismatch was not found to be a significant predictor of subsidence grade. However, the use of 18-mm-wide implants was associated with the development of significantly higher subsidence grade.

Based on the biomechanics of the lumbar spine, it is intuitive that utilizing the wider 22-mm implants would reduce the risk of high-grade subsidence. Grant et al. conducted a biomechanical study that demonstrated varying strength and stiffness across the lumbar and sacral endplates, with the center of the endplate being the weakest area. ${ }^{4}$ Another study of interbody fusion grafts determined that grafts covering at least $30 \%$ of the endplate had significantly higher resistance to subsidence than those covering only $25 \%$. $^{3}$ These findings offer support to using the wider 22-mm grafts in reducing the risk of highgrade subsidence by covering a larger area of the vertebral body. Moreover, the use of wider implant grafts can cover a larger surface area of the endplate and take advantage of the areas of regional strength near the periphery. A wider graft also has a larger surface area that can disperse axial loads across a larger area of the endplate. Previous studies have demonstrated high rates of endplate and implant mismatch for both lumbar and cervical implants. ${ }^{5,21}$ Future studies with larger sample sizes may prove necessary to show statistical significance.

Previous studies investigating cage subsidence after lumbar interbody fusion have also found more favorable outcomes with using wider implants. In a study of 140 patients and 238 levels fused in the lumbar spine, Le et al. demonstrated significantly higher rates of subsidence in patients with cages smaller than $18 \mathrm{~mm}$ (14.1\%) versus those with cages larger than $22 \mathrm{~mm}(1.9 \%) .{ }^{10}$ Another study investigated the use of even wider cages $(26 \mathrm{~mm})$ and found that these had significantly reduced cage settling compared to both the $18-\mathrm{mm}$ and $22-\mathrm{mm}$ cages, with a reduced loss of foraminal height when compared to the 18-mm cages. ${ }^{9}$ Finally, Marchi et al. demonstrated that use of the smaller 18-mm implants was significantly associated with higher subsidence grade. ${ }^{13}$ The current study demonstrated findings similar to previous reports that wider cages reduce the risk of high-grade subsidence. These results, in context of the current literature, suggest that larger implants should be utilized whenever feasible. 
TABLE 2. Severity of subsidence according to Marchi grade based on graft width and mean endplate-implant area mismatch

\begin{tabular}{ccccc}
\hline Marchi Grade & 18-mm Implant & 22-mm Implant & Superior Endplate Mismatch & Inferior Endplate Mismatch \\
\hline 0 & 1 & 5 & $2.69 \mathrm{~cm}^{2}$ & $1.86 \mathrm{~cm}^{2}$ \\
\hline I & 1 & 3 & $1.21 \mathrm{~cm}^{2}$ & $2.51 \mathrm{~cm}^{2}$ \\
\hline II & 2 & 10 & $1.47 \mathrm{~cm}^{2}$ & $1.81 \mathrm{~cm}^{2}$ \\
\hline III & 4 & 4 & $1.94 \mathrm{~cm}^{2}$ & $2.28 \mathrm{~cm}^{2}$ \\
\hline
\end{tabular}

The correlation between radiographic subsidence and clinical outcomes is still currently unclear. Settling of the implant can cause a reduction in disc height or foraminal height, resulting in a loss of indirect decompression and the potential for pain or neurological symptoms to return. However, the study by Le et al. observed only 3 patients who developed symptoms out of 20 with radiographic subsidence..$^{10}$ Additionally, another study investigating the effects of foraminal height and disc height subsidence on the development of radicular pain failed to find any significant correlations. ${ }^{2}$ Similar to those results, this study did not find any statistically significant relationship between Marchi grade and postoperative radiculopathy. The subsidence grade has been shown, however, to be a significant predictor of revision surgery for patients undergoing stand-alone LLIF. $^{20}$ The incidence for reoperation as a result of subsidence was $2.7 \%$ among all patients undergoing LLIF. ${ }^{12}$ The current study demonstrated higher reoperation rates for patients with $18-\mathrm{mm}$ implants $(62.5 \%)$ compared to those with 22-mm implants (56\%), but this did not reach statistical significance. Still, the current literature remains inconclusive, and to this end, this study is limited given its evaluation of radiographic subsidence. As such, further evaluation of the correlation between subsidence and clinical outcomes is warranted.

In addition to the width of an implant influencing graft subsidence, other operative factors such as endplate preparation have been indicated. Careful preparation of the endplate intraoperatively is essential to maintaining the structural integrity of the vertebral body. With the endplates removed, lumbar vertebral bodies only have $33 \%$ of the failure load compared to intact endplates..$^{15}$ During preparation of the endplates, extreme care should be taken to minimize destruction of cortical bone. Excessive loss of cortical bone exposes the weaker underlying cancellous bone that is less capable of resisting axial forces and subsidence of the graft. Comorbid osteoporosis or decreased bone mineral density affects subsidence in a similar way; weaker bones have reduced failure loads and facilitate the sinking of implants into the vertebral body. Impaired bone mineral density was found to be a major predictor of graft subsidence in LLIF and other surgical approaches with femoral neck t-scores less than $-1.0^{1,19}$ and adjacent vertebra t-scores less than -3.0. ${ }^{14}$ Subsidence is a multifactorial process influenced by both patient predispositions and surgical decision-making.

\section{Conclusions}

Among patients undergoing stand-alone LLIF in this cohort, the use of 18-mm-wide implants was associated with higher Marchi subsidence grade compared to the wider 22-mm implants. Mismatch between the endplate and implant area, length, or width was not significantly associated with a higher Marchi subsidence grade. Additionally, no significant relationship was found between either Marchi subsidence grade or endplate-implant mismatch and the presence of postoperative radiculopathy. Utilizing wider implants for LLIF, when feasible, can reduce the risk of high-grade subsidence that necessitates reoperation.

\section{Acknowledgments}

We express gratitude to Yue-Fang Chang, $\mathrm{PhD}$, for her invaluable assistance with statistical analysis of the data.

\section{References}

1. Agarwal N, Faramand A, Alan N, Tempel ZJ, Hamilton DK, Okonkwo DO, et al: Lateral lumbar interbody fusion in the elderly: a 10-year experience. J Neurosurg Spine 29:525529, 2018

2. Alimi M, Hofstetter CP, Cong GT, Tsiouris AJ, James AR, Paulo D, et al: Radiological and clinical outcomes following extreme lateral interbody fusion. J Neurosurg Spine 20:623-635, 2014

3. Closkey RF, Parsons JR, Lee CK, Blacksin MF, Zimmerman MC: Mechanics of interbody spinal fusion. Analysis of critical bone graft area. Spine (Phila Pa 1976) 18:1011-1015, 1993

4. Grant JP, Oxland TR, Dvorak MF: Mapping the structural properties of the lumbosacral vertebral endplates. Spine (Phila Pa 1976) 26:889-896, 2001

5. Gstoettner M, Heider D, Liebensteiner M, Bach CM: Footprint mismatch in lumbar total disc arthroplasty. Eur Spine J 17:1470-1475, 2008 (Erratum Eur Spine J 18:118, 2009)

6. Humphreys SC, Hodges SD, Patwardhan AG, Eck JC, Murphy RB, Covington LA: Comparison of posterior and transforaminal approaches to lumbar interbody fusion. Spine (Phila Pa 1976) 26:567-571, 2001

7. Kozak JA, Heilman AE, O'Brien JP: Anterior lumbar fusion options. Technique and graft materials. Clin Orthop Relat Res (300):45-51, 1994

8. Kwon B, Kim DH: Lateral lumbar interbody fusion: indications, outcomes, and complications. J Am Acad Orthop Surg 24:96-105, 2016

9. Lang G, Navarro-Ramirez R, Gandevia L, Hussain I, Nakhla J, Zubkov M, et al: Elimination of subsidence with 26-mmwide cages in extreme lateral interbody fusion. World Neurosurg 104:644-652, 2017

10. Le TV, Baaj AA, Dakwar E, Burkett CJ, Murray G, Smith DA, et al: Subsidence of polyetheretherketone intervertebral cages in minimally invasive lateral retroperitoneal transpsoas lumbar interbody fusion. Spine (Phila Pa 1976) 37:1268-1273, 2012 
11. Lehmen JA, Gerber EJ: MIS lateral spine surgery: a systematic literature review of complications, outcomes, and economics. Eur Spine J 24 (Suppl 3):287-313, 2015

12. Macki M, Anand SK, Surapaneni A, Park P, Chang V: Subsidence rates after lateral lumbar interbody fusion: a systematic review. World Neurosurg 122:599-606, 2019

13. Marchi L, Abdala N, Oliveira L, Amaral R, Coutinho E, Pimenta L: Radiographic and clinical evaluation of cage subsidence after stand-alone lateral interbody fusion. J Neurosurg Spine 19:110-118, 2013

14. Oh KW, Lee JH, Lee JH, Lee DY, Shim HJ: The correlation between cage subsidence, bone mineral density, and clinical results in posterior lumbar interbody fusion. Clin Spine Surg 30:E683-E689, 2017

15. Oxland TR, Grant JP, Dvorak MF, Fisher CG: Effects of endplate removal on the structural properties of the lower lumbar vertebral bodies. Spine (Phila Pa 1976) 28:771-777, 2003

16. Ozgur BM, Aryan HE, Pimenta L, Taylor WR: Extreme Lateral Interbody Fusion (XLIF): a novel surgical technique for anterior lumbar interbody fusion. Spine J 6:435-443, 2006

17. Rajaraman V, Vingan R, Roth P, Heary RF, Conklin L, Jacobs GB: Visceral and vascular complications resulting from anterior lumbar interbody fusion. J Neurosurg 91 (1 Suppl):60-64, 1999

18. Sasso RC, Kenneth Burkus J, LeHuec JC: Retrograde ejaculation after anterior lumbar interbody fusion: transperitoneal versus retroperitoneal exposure. Spine (Phila Pa 1976) 28:1023-1026, 2003

19. Tempel ZJ, Gandhoke GS, Okonkwo DO, Kanter AS: Impaired bone mineral density as a predictor of graft subsidence following minimally invasive transpsoas lateral lumbar interbody fusion. Eur Spine J 24 (Suppl 3):414-419, 2015
20. Tempel ZJ, McDowell MM, Panczykowski DM, Gandhoke GS, Hamilton DK, Okonkwo DO, et al: Graft subsidence as a predictor of revision surgery following stand-alone lateral lumbar interbody fusion. J Neurosurg Spine 28:50-56, 2018

21. Thaler M, Hartmann S, Gstöttner M, Lechner R, Gabl M, Bach C: Footprint mismatch in total cervical disc arthroplasty. Eur Spine J 22:759-765, 2013

\section{Disclosures}

Dr. Tempel reports being a consultant for NuVasive. Dr. Okonkwo reports being a consultant for NuVasive and Zimmer Biomet; he owns stock in Stryker. Dr. Kanter reports being a consultant for NuVasive; he receives royalties from Zimmer Biomet. Dr. Agarwal reports receiving royalties from Thieme Medical Publishers.

\section{Author Contributions}

Conception and design: Hamilton, Agarwal, Okonkwo, Kanter. Acquisition of data: Agarwal, Zhang, Tempel. Analysis and interpretation of data: Hamilton, Agarwal, White, Zhang, Okonkwo, Kanter. Drafting the article: Agarwal, White, Zhang. Critically revising the article: all authors. Reviewed submitted version of manuscript: all authors. Statistical analysis: Zhang. Administrative/technical/material support: Hamilton, Okonkwo, Kanter. Study supervision: Hamilton.

\section{Correspondence}

D. Kojo Hamilton: University of Pittsburgh Medical Center, Pittsburgh,PA.hamiltondk@upmc.edu. 